\title{
Chest X-ray patterns of pulmonary multidrug-resistant tuberculosis in children in a high HIV-prevalence setting
}

\begin{tabular}{|c|c|}
\hline \multicolumn{2}{|c|}{$\begin{array}{l}\text { Authors: } \\
\text { Samuel Manikkam }{ }^{1} \\
\text { Moherndran Archary }^{2} \\
\text { Raziya Bobat }^{2}\end{array}$} \\
\hline \multicolumn{2}{|c|}{$\begin{array}{l}\text { Affiliations: } \\
\text { Department of Radiology, } \\
\text { University of KwaZulu-Natal, } \\
\text { Durban, South Africa }\end{array}$} \\
\hline \multicolumn{2}{|c|}{$\begin{array}{l}{ }^{2} \text { Department of Paediatrics } \\
\text { and Child Health, University } \\
\text { of KwaZulu-Natal, Durban, } \\
\text { South Africa }\end{array}$} \\
\hline \multicolumn{2}{|c|}{$\begin{array}{l}\text { Corresponding author and } \\
\text { email: } \\
\text { Samuel Manikkam } \\
\text { samuel_m@webmail.co.za }\end{array}$} \\
\hline \multicolumn{2}{|c|}{$\begin{array}{l}\text { Postal address: } \\
\text { PO Box 1305, Wandsbeck, } \\
3631\end{array}$} \\
\hline \multicolumn{2}{|c|}{$\begin{array}{l}\text { Dates: } \\
\text { Received: } 22 \text { Apr. } 2015 \\
\text { Accepted: } 13 \text { Oct. } 2015 \\
\text { Published: } 18 \text { Mar. } 2016\end{array}$} \\
\hline \multicolumn{2}{|c|}{$\begin{array}{l}\text { How to cite this article: } \\
\text { Manikkam S., Archary M., } \\
\text { Bobat R. 'Chest X-ray patterns } \\
\text { of pulmonary multidrug- } \\
\text { resistant tuberculosis in } \\
\text { children in a high HIV- } \\
\text { prevalence setting' S Afr J } \\
\text { Rad. 2016;20(1); Art. \#829, } \\
6 \text { pages. http://dx.doi. } \\
\text { org/10.4102/sajr.v20i1.829 }\end{array}$} \\
\hline \multicolumn{2}{|c|}{$\begin{array}{l}\text { Copyright: } \\
\text { (c) 2016. The Authors. } \\
\text { Licensee: AOSIS. This work is } \\
\text { licensed under the Creative } \\
\text { Commons Attribution } \\
\text { License. }\end{array}$} \\
\hline \multicolumn{2}{|l|}{ Read online: } \\
\hline 口我和回 & $\begin{array}{l}\text { Scan this QR } \\
\text { code with your } \\
\text { smart phone or } \\
\text { mobile device } \\
\text { to read online. }\end{array}$ \\
\hline
\end{tabular}

Background: Paediatric multidrug-resistant tuberculosis (MDR-TB) necessitates a prolonged duration of treatment with an intensive treatment regimen. The chest X-ray patterns of pulmonary TB depend on a multiplicity of factors, including immune status, and therefore identifying the influence of HIV on the chest X-ray appearances of MDR-TB may assist with improving the diagnostic criteria.

Objectives: To describe the demographic characteristics and chest $X$-ray patterns of children with pulmonary MDR-TB and to compare the chest X-ray patterns of pulmonary MDR-TB between children who are HIV-infected and HIV-uninfected.

Method: Retrospective chart review of hospital notes and chest X-rays of children with pulmonary MDR-TB at King Dinuzulu Hospital, Durban. The chest X-rays were systematically reviewed for the presence of the following variables: hilar/mediastinal lymphadenopathy, bronchopneumonic opacification, segmental/lobar consolidation, cavities, miliary opacification and pleural effusion.

Results: Forty-five children (mean age, 6.29 years; median age, 6.00 years) with pulmonary MDR-TB met the inclusion criteria. The most common chest X-ray finding was consolidation (53.5\%), followed by lymphadenopathy (35.6\%), bronchopneumonic opacification (33.3\%) and cavities (31.1\%). Cavities were more common (OR 6.1; 95\% CI 1.52-24.66) in children who had been initiated on standard anti-TB treatment for the current TB episode. There were no statistically significant differences in any of the chest X-ray patterns in HIV-uninfected $(n=22)$ compared with HIV-infected $(n=20)$ children.

Conclusion: The most common chest X-ray finding was consolidation, followed by lymphadenopathy, bronchopneumonic opacification and cavities. The finding of a significantly higher frequency of cavities in children who had received prior standard anti-TB treatment for the current TB episode could reflect poor disease containment and increased parenchymal damage, owing to a delay in the recognition of MDR-TB. The development of cavitation in chest X-rays of children with TB could raise concern for the possibility of MDR-TB, and prompt further testing.

\section{Introduction}

Globally, paediatric tuberculosis (TB) remains an area of significant concern. In 2012, the total estimated number of childhood notifications from all countries was $349000 .{ }^{1}$ In the paediatric population, chest $X$-rays remain the initial investigation in the work-up of suspected pulmonary TB; however, overlapping clinical and chest X-ray features with other causes of acute pneumonia, and poor sensitivities for the tuberculin skin test (TST), make confirmation of TB difficult. ${ }^{2}$ The emergence of multidrug-resistant TB (MDR-TB), defined as resistance to both isoniazid and rifampicin, is a particular area of public health concern in South Africa, where the estimated MDR-TB burden in 2012 was 1.8\% for new cases and $6.7 \%$ for retreatment cases, with a total number of laboratory-confirmed MDR-TB cases of $15419 .{ }^{1}$

Drug-resistant TB necessitates a prolonged duration of treatment with a more intensive treatment regimen. In terms of the clinical outcome of paediatric MDR-TB, in a cohort study of children with culture-confirmed MDR-TB in the Western Cape, South Africa, the majority of children were treated successfully, with $82 \%$ having favourable outcomes (cured and treatment completed) in contrast to adult data. ${ }^{3}$ Although the majority of children were treated successfully, a further study in this region, which documented four known deaths $(10 \%)$, suggested that earlier recognition of drug resistance could probably have prevented a further two deaths and morbidity. ${ }^{4}$ 
This observation highlights the importance of early detection and treatment of MDR-TB.

In poorly resourced settings, drug-susceptibility testing is frequently reserved for cases that do not respond to standard anti-TB treatment. Early identification of MDR-TB with early onset of treatment may prevent morbidity and mortality; therefore identifying chest $\mathrm{X}$-ray features which may alert the clinician to the possibility of MDR-TB on initial presentation may be important. The most commonly described chest $X$-ray findings in children with MDR-TB are segmental/lobar opacification $(70 \%)^{3,4}$ and hilar/mediastinal lymphadenopathy $(48 \%-51 \%){ }^{3,4}$ Cavities have been described in $35 \%-38 \%{ }^{3,4}$

The chest X-ray manifestations of pulmonary TB depend on several factors, including age and immune status, ${ }^{5}$ therefore identifying the influence of $\mathrm{HIV}$ on the chest X-ray appearances of MDR-TB may be of value. To our knowledge, no studies have compared the chest $\mathrm{X}$-ray appearance of pulmonary MDR-TB between HIV-infected and HIVuninfected children.

\section{Objectives}

The objectives of the study were to:

1. describe the demographic characteristics of children with pulmonary MDR-TB

2. describe the chest X-ray patterns of children with pulmonary MDR-TB

3. compare the chest X-ray patterns of pulmonary MDR-TB between children who are HIV-infected and HIVuninfected.

\section{Ethical clearances}

Ethical approval was obtained from the University of KwaZulu-Natal Biomedical Research Ethics Committee and site approval was obtained from the hospital. Informed consent was not required for the retrospective study.

\section{Method}

A retrospective review of patient records was conducted at the paediatric ward and paediatric outpatients' department of a referral MDR-TB facility in Durban (King Dinuzulu Hospital) for the period 01 January 2013 to 31 December 2013, to identify children diagnosed with pulmonary MDR-TB.

Children ( $<12$ years) were included if they had been diagnosed with pulmonary MDR-TB between 01 January 2013 and 31 December 2013, and a baseline chest X-ray performed within one week of initiation of MDR-TB treatment.

MDR-TB (defined as resistance to both isoniazid and rifampicin) was diagnosed on samples of sputum, gastric aspirate or bronchoalveolar lavage using the following methods:

1. molecular testing using polymerase chain reaction (PCR) with MTBDRplus, a molecular genetic assay for identification of resistance to rifampicin and/or isoniazid of Mycobacterium tuberculosis complex

2. agar-based culture and drug sensitivity testing

3. If the results for the above methods were not available, rifampicin resistance was confirmed with molecular testing using Gene Xpert.

Data were collected through chart review. The following information was extracted from the available records:

- results of drug-susceptibility testing

- history of contact with an adult with pulmonary TB, obtained through primary caregiver recall (as recorded in chart notes)

- duration of any previous anti-TB treatment for the current episode. This was defined as a standard treatment regimen (i.e. isoniazid, rifampicin and pyrazinamide, with or without ethambutol) and did not include TB prophylaxis.

- history of a previous TB episode, which was defined as standard TB treatment for at least 1 month, followed by a symptom-free period of at least 6 months (determined on the basis of reports by the parent/caregiver) before the start of the current MDR-TB episode; this is a commonly used programmatic definition of a separate episode ${ }^{3}$

- HIV status. In HIV-infected children, the following additional information was obtained from the patient records: antiretroviral (ARV) treatment regimen, and absolute CD4 T-lymphocyte count and percentage, at the time of the baseline chest $\mathrm{X}$-ray. In this group of children, the degree of immunosuppression was stratified, depending on the CD4 count, using the WHO immunological classification. ${ }^{6}$

Frontal chest $\mathrm{X}$-rays were acquired using digital radiography (with Kodak dual or single receptor units DR 7500 and 9500 respectively) or analogue radiography (with a Picker Synergen 350 unit). The images were printed to make film hard copies. The chest $X$-rays were all read by the same senior consultant radiologist, who read chest $\mathrm{X}$-rays systematically with a standardised recording. The reporting radiologist was blinded to the HIV status and immunological data.

The chest X-rays were reviewed for the presence of the following variables:

- hilar/mediastinal lymphadenopathy, described as nodular masses according to site ${ }^{7}$

- bronchopneumonic opacification, described as multiple, ill-defined, confluent, nodular opacities ${ }^{8}$

- segmental/lobar consolidation, described as a homogeneous increase in pulmonary parenchymal attenuation that obscures the margins of vessels and airway walls ${ }^{9}$

- cavities, described as gas-filled spaces, seen as a lucency or low-attenuation area, within pulmonary consolidation ${ }^{9}$

- miliary opacification, described as profuse, tiny, discrete, rounded pulmonary opacities ( $\leq 3 \mathrm{~mm}$ in diameter) that are generally uniform in size and diffusely distributed throughout the lungs ${ }^{9}$

- $\quad$ pleural effusion, described as pleural opacification. ${ }^{7}$ 
Statistical analysis was performed using IBM SPSS statistics software. For continuous variables such as age, the mean, median and range were calculated. Results were recorded as frequencies and percentages for categorical variables. The Pearson chi-square test or Fisher exact test (where cell frequencies were $<5$ ) were used to test for significant relationships between the frequency of each chest X-ray pattern, and the age. The level of significance was set at $p<0.05$. The odds ratio (OR) and $95 \%$ confidence limits (CI) were estimated using univariate logistic regression in SPSS.

\section{Results}

During the study period, 55 children $<12$ years were diagnosed with pulmonary MDR-TB based on clinical and laboratory findings; however, 10 children were excluded from this analysis as they did not have a retrievable chest X-ray taken within 1 week of MDR-TB treatment initiation; therefore the chest $X$-rays of 45 children were analysed in the present study (Figure 1). The median age was 6.00 years, and age range 3 months to 11 years and 9 months. Twenty-two children (48.9\%) were HIV uninfected, and 20 children (44.4\%) were HIV infected. The HIV status was unknown for 3 children (Figure 1). In the 20 children who were HIV infected, $16(80 \%)$ were on an ARV treatment regimen. MDR-TB was confirmed using specimens of sputum (19 children [42.2\%]), gastric aspirate (14 children [31.1\%]) and bronchoalveolar lavage (1 child [2.2\%]), and was unspecified in 11 children (Figure 2 and Figure 3).

Eighteen children (40\%) had a history of contact with an adult with $\mathrm{TB}$, of whom 8 children reported a history of a contact diagnosed with MDR-TB; and 6 children indicated no prior TB treatment for the current episode.

Prior to presentation at the MDR-TB facility, 19 children $(42.2 \%)$ had been initiated on a standard anti-TB treatment regimen for the current TB episode. In this group, there was a mean duration of 4.39 months (range 0.25 - 12 months) of treatment prior to a diagnosis of MDR-TB being made.

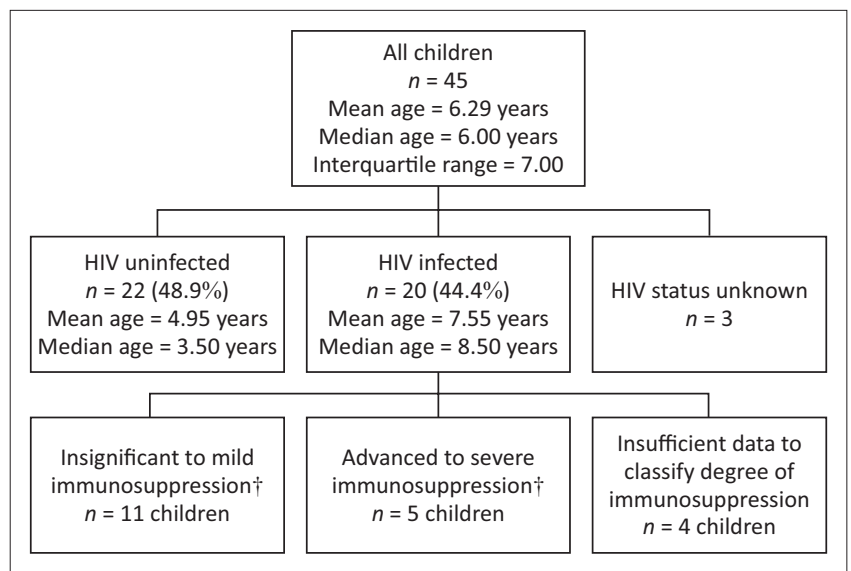

$\dagger$, World Health Organization. WHO case definitions of HIV for surveillance and revised clinical staging and immunological classification of HIV-related disease in adults and children. 2007 [cited 11 September 2013]. Available from: http://www.who.int/hiv/ pub/guidelines/HIVstaging150307.pdf

FIGURE 1: Demographics.
Sixteen children (35.6\%) were immediately initiated on MDR$\mathrm{TB}$ treatment following diagnosis. In 10 children (22.2\%), no history of an anti-TB treatment regimen for the current TB episode was recorded. A history of a prior episode of TB was found in 13 children (28.9\%), of whom only 1 child had a reported history of previous MDR-TB.

On evaluation of the chest $X$-ray patterns, 22 children (48.9\%) had $\geq 2$ findings on the chest $X$-ray, and $10(22.2 \%)$ had no

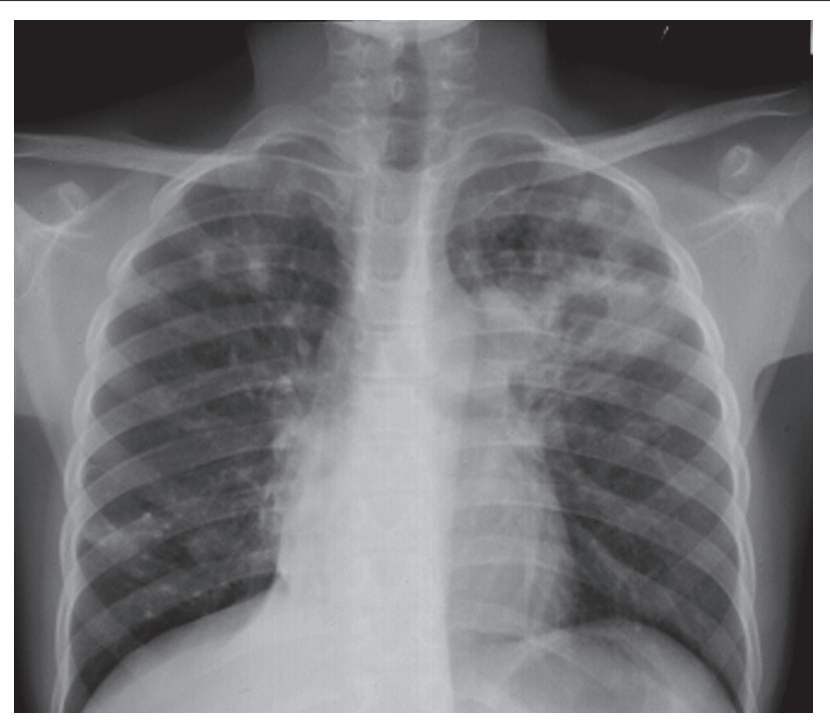

FIGURE 2: Pulmonary multidrug-resistant tuberculosis in an 11-year-old girl. Frontal chest radiograph. Bronchopneumonic opacification and consolidation with cavitation is noted in the left upper lobe. III-defined focal consolidation with cavitation is evident in the right upper zone. Dense nodular opacities are noted in the right lower zone, suggesting calcified granulomas. Aortopulmonary window and right hilar lymphadenopathy is noted. Evidence of right lower lobe atelectasis.

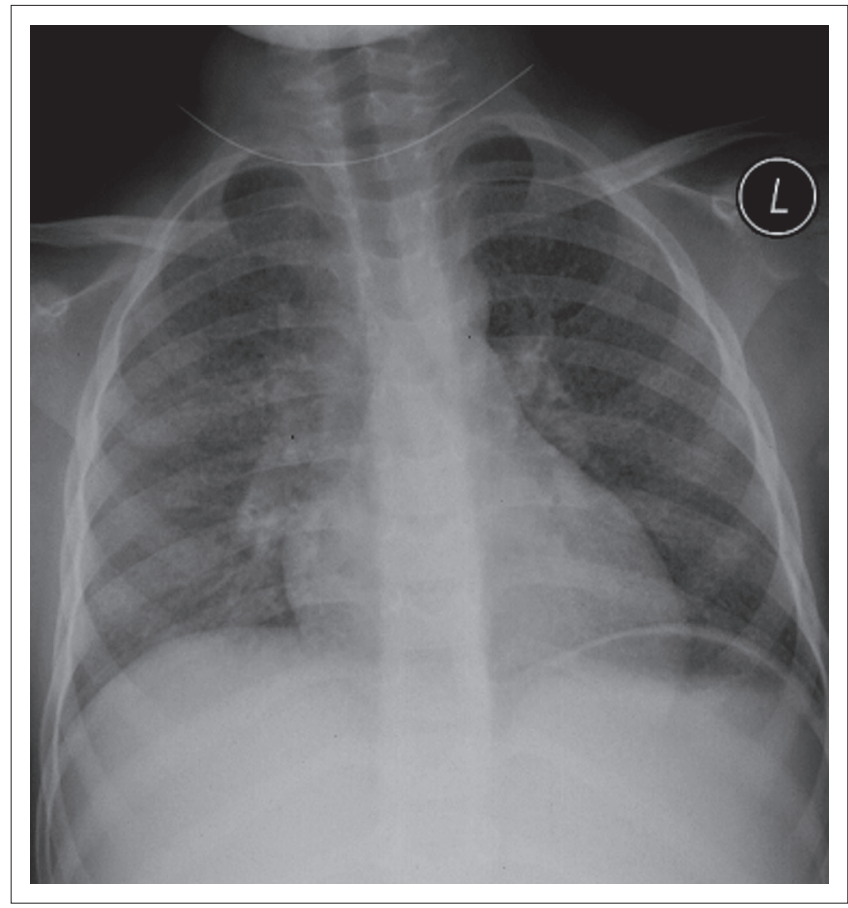

FIGURE 3: Multidrug-resistant tuberculosis in a 6-year-old girl. Frontal chest radiograph demonstrating diffuse miliary opacification. There is enlargement of the hilar regions bilaterally suggestive of associated hilar lymphadenopathy. 
abnormality. The most common chest X-ray finding in all children was segmental/lobar consolidation (53.5\%), followed by lymphadenopathy (35.6\%), bronchopneumonic opacification (33.3\%) and cavities (31.1\%). When comparing the chest $X$-ray patterns in children of ages $0-6$ years and $6-12$ years, miliary opacification and pleural effusions were not seen in the $0-6$-year age group, and cavities were found more frequently in the $6-12$-year age group $(40.7 \%$ in older children compared with $16.7 \%$ in younger children) (Table 1 ).

Cavities were found more frequently in children who had been initiated on a standard anti-TB treatment regimen for the current TB episode, compared with children with no recorded history of standard anti-TB treatment for the current episode (52.6\% compared with 15.4\%; OR 6.111 (1.515-24.658) (Table 2).

Chest X-ray findings of bronchopneumonic opacification, segmental/lobar consolidation, cavities and miliary opacification were common in HIV-infected children compared with HIV-uninfected children; however, this was not statistically significant (Table 3).

\section{Discussion}

MDR-TB represents a significant challenge in the attainment of global TB control. The identification of this clinical entity is equally important in the paediatric population, because a child with TB was shown to be as likely as an adult to have MDR-TB. ${ }^{1}$ In clinical practice, early identification is important as it will result in a reduction of morbidity and mortality relating to the disease. Factors contributing to early identification include chest $\mathrm{X}$-ray changes as well as a history of contact.

The most frequent chest X-ray finding in children with pulmonary MDR-TB in our study was consolidation, followed by lymphadenopathy, bronchopneumonic opacification and cavities. When comparing the chest X-ray patterns in the different age groups, pleural effusions were not seen in the 0 - 6-year age group. This is consistent with the literature which described tuberculous pleural effusion developing mainly in children $>5$ years old following recent primary infection. ${ }^{10}$ Cavities were found more frequently in the older age group (6-12 years), consistent with adult-type cavitating disease, which is the dominant disease manifestation following recent primary infection in older children ( $>10$ years of age).$^{10}$

The finding of a significantly higher frequency of cavities in children who had received prior standard anti-TB treatment for the current TB episode, compared with children with no recorded history of standard anti-TB treatment for the current episode, may reflect poor disease containment and increased parenchymal damage, ${ }^{10}$ either owing to a delay in the recognition of MDR-TB, or the development of MDR-TB owing to an inappropriate treatment regimen or poor compliance with the standard treatment regimen, although the development of MDR-TB may be a less likely consideration because of the paucibacillary nature of paediatric TB. ${ }^{4}$

Regarding the effect of drug resistance on the chest X-ray appearance of pulmonary $\mathrm{TB}$, we compared our findings with studies by Donald et al. ${ }^{11}$ and Lamont et al. ${ }^{7}$ of children

TABLE 1: Chest X-ray patterns in all children and comparison by age.

\begin{tabular}{|c|c|c|c|c|}
\hline Chest X-ray pattern & $\begin{array}{l}\text { All children } \\
(\%)(n=45)\end{array}$ & $\begin{array}{l}\text { Children age } 0-6 \text { years } \\
(\%)(n=18)\end{array}$ & $\begin{array}{c}\text { Children age } 6-12 \text { years } \\
(\%)(n=27)\end{array}$ & $\begin{array}{l}p \text { value in comparing children age } 0-6 \\
\text { years and } 6-12 \text { years }\end{array}$ \\
\hline Hilar/mediastinal lymphadenopathy & $16(35.6)$ & $5(27.8)$ & $11(40.7)$ & 0.373 \\
\hline Bronchopneumonic opacification & $15(33.3)$ & $5(27.8)$ & $10(37.0)$ & 0.519 \\
\hline Segmental/lobar consolidation & $24(53.5)$ & $9(50.0)$ & $15(55.6)$ & 0.714 \\
\hline Cavities & $14(31.1)$ & $3(16.7)$ & $11(40.7)$ & 0.087 \\
\hline Miliary opacification & $6(13.3)$ & 0 & $6(22.2)$ & - \\
\hline Pleural effusion & $5(11.1)$ & 0 & $5(18.5)$ & - \\
\hline
\end{tabular}

TABLE 2: Comparison of chest X-ray patterns in children with prior standard TB treatment for the current episode and children with no recorded prior standard TB treatment for the current episode.

\begin{tabular}{|c|c|c|c|}
\hline Chest X-ray pattern & Prior standard TB treatment $(\%)(n=19)$ & No recorded prior TB treatment $(\%)(n=26)$ & Odds ratio $(95 \% \mathrm{Cl})$ \\
\hline Hilar/mediastinal lymphadenopathy & $7(36.8)$ & $9(34.6)$ & $1.102(0.321-3.783)$ \\
\hline Bronchopneumonic opacification & $8(42.1)$ & $7(26.9)$ & $1.974(0.562-6.939)$ \\
\hline Segmental/lobar consolidation & $12(63.2)$ & $12(46.2)$ & $2.000(0.597-6.705)$ \\
\hline Cavities & $10(52.6)$ & $4(15.4)$ & $6.111(1.515-24.658)$ \\
\hline Miliary opacification & $3(15.8)$ & $3(11.5)$ & $1.437(0.257-8.050)$ \\
\hline Pleural effusion & $2(10.5)$ & $3(11.5)$ & $0.902(0.135-6.005)$ \\
\hline
\end{tabular}

TABLE 3: Comparison of chest X-ray patterns in HIV-uninfected and HIV-infected children.

\begin{tabular}{|c|c|c|c|}
\hline Chest X-ray pattern & HIV uninfected $(\%)(n=22)$ & HIV infected $(\%)(n=20)$ & Odds ratio $(95 \% \mathrm{Cl})$ \\
\hline Hilar/mediastinal lymphadenopathy & $9(40.9)$ & $7(35.0)$ & $0.558(0.218-1.428)$ \\
\hline Bronchopneumonic opacification & $6(27.3)$ & $9(45.0)$ & $0.877(0.422-1.823)$ \\
\hline Segmental/lobar consolidation & $11(50.0)$ & $12(60.0)$ & $1.000(0.520-1.925)$ \\
\hline Cavities & $7(31.8)$ & $7(35.0)$ & $0.697(0.294-1.651)$ \\
\hline Miliary opacification & $3(13.6)$ & $3(15.0)$ & $0.742(0.223-2.471)$ \\
\hline Pleural effusion & $3(13.6)$ & $2(10.0)$ & $0.573(0.121-2.717)$ \\
\hline Normal & $4(18.2)$ & $4(20.0)$ & - \\
\hline
\end{tabular}


in the Western Cape with bacteriologically confirmed pulmonary TB. Although these study populations were not tested for MDR-TB, the incidence is presumed to be low because MDR-TB was first detected in South Africa in the mid-1980s, with subsequent surveillance data for this period showing low prevalence levels. ${ }^{12}$ Our study found a higher frequency of cavitation $\left(31.3 \%\right.$ v. $\left.14.0 \%{ }^{11}-16.2 \%{ }^{7}\right)$. The finding of a higher frequency of cavitation in MDR-TB reflects a similar trend in adult patients, where cavities were more frequently observed in computed tomography (CT) scans of MDR-TB adult patients as compared with drug sensitive (DS)-TB; ${ }^{13}$ and multiple cavities were seen more frequently in MDR-TB adult patients as compared with DS-TB on both chest $\mathrm{X}$-rays ${ }^{13}$ and $\mathrm{CT} .{ }^{14}$ The development of cavitation in chest $\mathrm{X}$-rays of children with TB could raise concern for the possibility of MDR-TB, and prompt further testing.

When compared with the studies of Donald et al. ${ }^{11}$ and Lamont et al., ${ }^{7}$ our study found a lower frequency of lymphadenopathy $\left(35.6 \%\right.$ v. $\left.62.5 \%{ }^{11}-63.0 \%{ }^{7}\right)$ that could be partly explained by the absence of lateral chest X-rays in our study, which are of value in detecting lymphadenopathy in children investigated for TB. ${ }^{15}$ Although our study found a lower frequency of lymphadenopathy when compared with the studies of Donald et al. ${ }^{11}$ and Lamont et al., lymphadenopathy was the second most common finding in our study, and is considered the radiological hallmark of primary TB in childhood. ${ }^{16}$ Therefore the combination of consolidation, cavitation and lymphadenopathy may alert the clinician to the possibility of MDR-TB and prompt further investigation.

The frequency of the rest of the chest X-ray findings in the study by Donald et al. were similar to those of our study: segmental lesion, referring to any radiographic opacity which filled a lobe or a segment of a lobe (56\%); bronchopneumonia $(30 \%)$; pleural effusion $(16 \%)$ and a miliary pattern $(10 \%) .{ }^{11}$

When comparing the frequency of chest X-ray patterns in HIV-uninfected and HIV-infected children, bronchopneumonic opacification was found more commonly in the HIV-infected group. Although not statistically significant, this may be the result of poor disease containment at the site of the Ghon focus or lymphadenopathy, owing to the immunosuppressive effect of HIV and subsequent intra-bronchial spread of disease. ${ }^{10}$ The absence of statistically significant differences between HIV-uninfected and HIV-infected children is consistent with a study evaluating the clinical features and outcome of children admitted to a TB hospital in the Western Cape in South Africa, where no statistically significant difference in chest X-ray appearances of TB was seen between these subgroups; however, in that study, drug resistance was confirmed in $19 \%$ of the 93 children for whom these test results were available. ${ }^{17}$

The chest X-ray patterns of children with pulmonary MDRTB described in our study are similar to the results of studies describing the clinical features of children with MDR-TB by Seddon et al. ${ }^{3}$ and Schaaf et al.; ${ }^{4}$ however, bronchopneumonic opacification was found more frequently in our study than in those by Seddon et al. ${ }^{3}$ and Schaaf et al. ${ }^{4}$ (33\% v. 19\%). The difference may be explained partly by the fact that the studies by Seddon et al. ${ }^{3}$ and Schaaf et al. ${ }^{4}$ included children with extrapulmonary TB as well as pulmonary TB. A further reason may be the limitation of the classification of bronchopneumonic opacification to describe widespread bronchopneumonic changes in the study by Seddon et al. ${ }^{3}$

\section{Limitations}

The limitations of the present study include its retrospective study design. A limitation in obtaining a history of a TB contact was that the history was based on recall of the parent or caregiver present at the first visit; in some cases, there might have been a TB contact that was not diagnosed at this time, and the actual figure might be even higher. The X-rays were read by a single radiologist only; therefore interobserver variability could not be determined, reducing the reliability of results. The presence of a viral or bacterial co-infection might have accounted for the chest X-ray appearances in certain cases. Missing CD4 counts and the sample size limited our ability to compare the influence of degree of immunosuppression owing to HIV on the chest X-ray patterns.

\section{Conclusion}

The most common chest X-ray finding in children with MDR-TB in our study was consolidation, followed by lymphadenopathy, bronchopneumonic opacification and cavities. Cavities were found more frequently in children who had received standard anti-TB treatment for the current TB episode, which may reflect poor disease containment and increased parenchymal damage owing to delay in the recognition of MDR-TB. The chest X-ray findings of consolidation, cavitation and lymphadenopathy on initial presentation, or the development of cavitation in chest $\mathrm{X}$-rays of children receiving standard anti-TB treatment, could raise concern for the possibility of MDR-TB and prompt further testing. We intend to pursue research with the inclusion of children with DS-TB as controls for further assessment. There were no statistically significant differences between patterns in HIV-uninfected and -infected children.

\section{Acknowledgements}

The authors thank Dr J.M. Kallideen and the management and staff of King Dinuzulu Hospital for their assistance; Fikile Nkwanyana, biostatistician at the University of KwaZulu-Natal, for statistical analysis of the data; and Leora Sewnarain, University of KwaZulu-Natal, for assistance with the editorial preparation of the manuscript.

\section{Competing interests}

The authors declare that they have no financial or personal relationships which may have inappropriately influenced them in writing this article. 


\section{Authors' contributions}

S.A.M. (University of KwaZulu-Natal) was the principal investigator and prepared the manuscript. R.B. (University of KwaZulu-Natal) and M.A. (University of KwaZulu-Natal) made conceptual contributions and were involved with the manuscript editing, and are respectively the supervisor and co-supervisor of S.A.M.'s Master's research.

\section{References}

1. World Health Organization. Global tuberculosis report 2013. c2013 [cited 22 March 2014]. Available from: http://apps.who.int/iris/bitstream/10665/91355/ 1/9789241564656_eng.pdf

2. Jeena PM. The interaction of HIV and tuberculosis in childhood. S Afr Med J. 2007;97:989-991.

3. Seddon JA, Hesseling AC, Willemse M, Donald PR, Schaaf HS. Culture-confirmed multidrug-resistant tuberculosis in children: clinical features, treatment, and outcome. Clin Infect Dis. 2012;54:157-166. http://dx.doi.org/10.1093/cid/cir772

4. Schaaf HS, Shean K, Donald PR. Culture confirmed multidrug resistant tuberculosis: diagnostic delay, clinical features, and outcome. Arch Dis Child. 2003;88 1106-1111. http://dx.doi.org/10.1136/adc.88.12.1106

5. Leung AN. Pulmonary tuberculosis: The essentials. Radiology. 1999;210:307-322. http://dx.doi.org/10.1148/radiology.210.2.r99ja34307

6. World Health Organization. WHO case definitions of HIV for surveillance and revised clinical staging and immunolgical classification of HIV-related disease in adults and children. c2007 [cited 17 August 2013]. Available from: http://www. who.int/hiv/pub/guidelines/HIVstaging150307.pdf
7. Lamont AC, Cremin BJ, Pelteret RM. Radiological patterns of pulmonary tuberculosis in the paediatric age group. Pediatr Radiol. 1986;16:2-7. http://dx. doi.org/10.1007/BF02387495

8. Lange S. Radiology of chest diseases. 3rd ed. Stuttgart: Georg Thieme Verlag; 2007. http://dx.doi.org/10.1055/b-002-59222

9. Hansell DM, Bankier AA, MacMahon H, McLoud TC, Muller NL, Remy J. Fleischner Society: Glossary of terms for thoracic imaging. Radiology. 2008;246:697-722. http://dx.doi.org/10.1148/radiol.2462070712

10. Marais BJ, Gie RP, Schaaf HS, et al. A proposed radiological classification of childhood intra-thoracic tuberculosis. Pediatr Radiol. 2004;34:886-894. http:// dx.doi.org/10.1007/s00247-004-1238-0

11. Donald PR, Ball JB, Burger PJ. Bacteriologically confirmed pulmonary tuberculosis in childhood. Clinical and radiological features. S Afr Med J. 1985;67:588-590

12. Weyer K, Brand J, Lancaster J, Levin J, Van der Walt M. Determinants of multidrug resistant tuberculosis in South Africa: Results from a national survey. S Afr Med J. 2007;9:1120-http://dx.doi.org/10.1148/radiol.24620707121128

13. Cha J, Lee HY, Lee KS, et al. Radiological findings of extensively drug-resistant pulmonary tuberculosis in non-AIDS adults: Comparisons with findings of multidrug-resistant and drug-sensitive tuberculosis. Korean J Radiol. 2009;10: 207-216. http://dx.doi.org/10.3348/kjr.2009.10.3.207

14. Myung Jin C, Kyung Soo L, Won-Jung K, et al. Drug-sensitive tuberculosis, multidrug-resistant tuberculosis, and nontuberculous mycobacterial pulmonary disease in nonAIDS adults: Comparisons of thin-section CT findings. Eur Radiol. 2006;16:1934-1941. http://dx.doi.org/10.1007/s00330-006-0174-9

15. Smuts NA, Beyers N, Gie RP, et al. Value of the lateral chest radiograph in tuberculosis in children. Pediatr Radiol. 1994;24:478-480. http://dx.doi.org/10. 1007/BF02015003

16. Leung AN, Müller NL, Pineda PR, FitzGerald JM. Primary tuberculosis in childhood Radiographic manifestations. Radiology. 1992;182:87-91. http://dx.doi.org/10. 1148/radiology.182.1.1727316

17. Soeters M, de Vries AM, Kimpen JL, Donald PR, Schaaf HS. Clinical features and outcome in children admitted to a TB hospital in the Western Cape--the influence of HIV infection and drug resistance. S Afr Med J. 2005;95:602-606. 